\title{
Evaluation of the Dissipative and Thermal Characteristics of a Magneto-Rheological (MR) Damper for Automotive Suspension
}

\author{
Barenten Suciu ${ }^{\mathrm{a},}{ }^{,}$, Keisuke Koyanagi ${ }^{\mathrm{b}}$, Keita Sonoda ${ }^{\mathrm{a}}$ \\ ${ }^{a}$ Department of Intelligent Mechanical Engineering, Fukuoka Institute of Technology, \\ 3-30-1 Wajiro-Higashi, Higashi-ku, Fukuoka 811-0295, Japan \\ ${ }^{\mathrm{b}}$ Graduate School of Engineering, Fukuoka Institute of Technology, \\ 3-30-1 Wajiro-Higashi, Higashi-ku, Fukuoka 811-0295, Japan \\ *Corresponding Author: suciu@fit.ac.jp
}

\begin{abstract}
In this work one evaluates the dissipative and thermal properties of a monotube long stroke magneto-rheological (MR) damper. Exposing the MR fluid to magnetic fields controllable via low input voltage, an intelligent automotive suspension can be achieved. Employing a ball-screw shaker, vibration and endurance tests of the MR damper are performed, and variation of the dissipated energy as well as the damping coefficient versus various working parameters is clarified. Based on a thermographical image processing technique, temperature distributions on the external surface of the MR damper are recorded during its forced heating under excitation tests, and variation of the generated heat versus working time is determined. Such results are useful for the appropriate design of automotive suspensions.
\end{abstract}

Keywords: intelligent suspension, thermographical image processing, dissipated energy and generated heat.

\section{Introduction}

Recently, manufactures have shown increased interest in using magneto-rheological (MR) dampers for automotive suspension. Such increase in the commercial interest can be attributed to the success in reducing the production costs of MR dampers. Thus, compared to other types of semi-active dampers, such as solenoid valves hydraulic dampers and electro-rheological (ER) dampers, nowadays commercially available MR dampers are about 10 times cheaper.

A classical hydraulic damper can be rendered as controllable under magnetic fields if iron micro-particles coated with an anti-coagulant material are dispersed in oil to produce a functional colloid of variable viscosity. When the MR fluid is exposed to a magnetic field controllable via a low variable voltage, the iron particles align themselves along the magnetic flux lines into very thin strings stretched from one magnetic pole to the other and perpendicular to each paramagnetic pole surface. When such MR fluid flows through orifices and valves, classically used in construction of hydraulic dampers, its apparent viscosity is controlled via a variable magnetic field. Even though the carrier fluid exhibits no change in viscosity, the MR colloid, as a whole, will appear to have undergone a change in viscosity.

MR dampers are also very attractive for developing intelligent automotive suspensions ${ }^{(1)}$, which require continuous monitoring of the rough road condition, by using onboard cameras, and suspensions incorporating absorbers which can be continuously controlled via a small power source. Such intelligent suspensions are collecting data at a speed of about 25 inputs per millisecond, and are able to provide real-time damping coefficient adjustment to avoid the tire from dropping at the bottom of a pothole, etc.

Although the MR damper manufacturer provides some technical data (linear variation of the damping force against the piston speed for various values of the control voltage), such data might be insufficient for the appropriate design of automotive suspensions. In this experimental work, the dissipative and thermal characteristics of a monotube long stroke MR damper are evaluated. Thus, by employing a made-in-house ball-screw shaker, vibration and endurance tests of the MR damper are performed. As a result, variation of the dissipated energy and damping coefficient versus various working parameters is clarified. Additionally, using a thermographical method ${ }^{(2,3)}$, temperature distributions on 
the external surface of the MR damper are recorded during its forced heating under excitation tests, followed by its natural cooling in air. As a result, variation of the generated heat versus the working time is also clarified.

\section{Test Rig and Experimental Procedure}

\subsection{Employed MR Damper}

In this experimental work, the dissipative and thermal characteristics of a monotube long stroke MR damper (LORD RD-8041-1 ${ }^{(4)}$ ), pre-charged through high-pressure nitrogen gas, are evaluated.

Characteristics of the tested MR damper are as follows: maximum cylinder diameter of $42 \mathrm{~mm}$, piston-rod diameter of $10 \mathrm{~mm}$, maximum stroke of $75 \mathrm{~mm}$, maximum (extended) length of $248 \mathrm{~mm}$, maximum damping force of $2.5 \mathrm{kN}$ (both in tension and compression), maximum piston speed of $0.2 \mathrm{~m} / \mathrm{s}$, and maximum operating temperature of $71{ }^{0} \mathrm{C}$.

In order to control the MR damper a LORD "Wonder Box Device Kit" (RD-3002-03 ${ }^{(5)}$ ) was employed, for a maximum input current of $1 \mathrm{~A}$, and the following discrete values of the control voltage: $0,0.4,0.8,1.2,1.6$ and $2.0 \mathrm{~V}$ (DC).

\section{$2.2 \quad$ Test Rig}

Test rig used during vibration and endurance experiments of the MR damper is shown in Fig. 1. The made-in-house ballscrew shaker consists of a ball-screw mechanism, driven by an electrical motor via belt transmission, a controller, and a power unit (Fig. 1). Frequency of the cyclical horizontal movement of the piston was set to $f=0.85 \mathrm{~Hz}$, equaling the natural frequency of the considered automotive suspension. However, actual (experimentally obtained) variation of the frequency versus the rotation speed $n$ of the ball-screw is illustrated in Fig. 2. One observes that excitation frequency linearly decreases for $n$ exceeding $10 \mathrm{~Hz}$, and this effect is induced by the inevitable lag of ramp-like variation of the compression-extension speed during an excitation period.

Endurance tests on the MR damper were performed for a time interval of 1 hour, under the following procedure (see Fig. 3 for the attached loading and measurement equipment). MR damper is fixed on the test rig and then an anti-reflection cover is installed, to increase the accuracy of the thermographical results by reducing the reflexivity of electromagnetic waves on the damper's external surface. Rotation speed $n$ is set to achieve the desired stroke $S$ (Fig. 4).

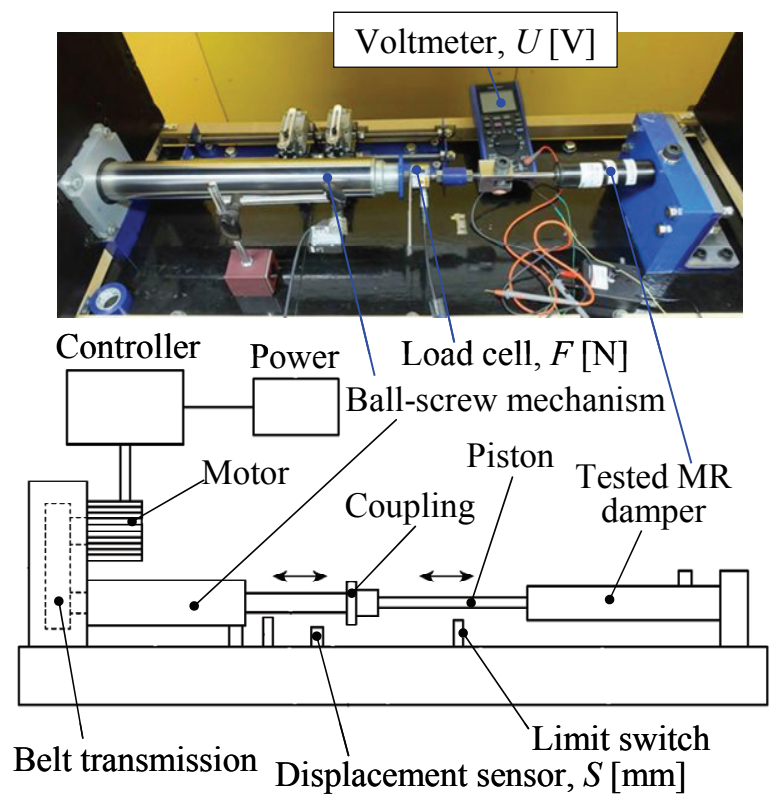

Fig. 1. Test rig used during vibration and endurance tests of the MR damper.

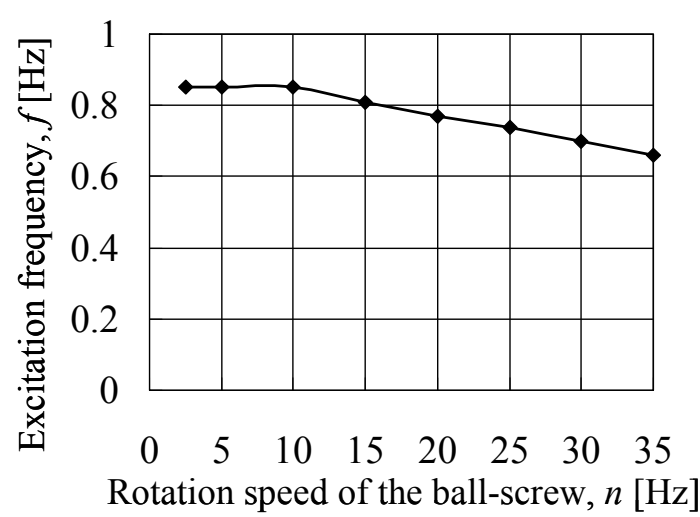

Fig. 2. Variation of the excitation frequency versus the rotation speed of the ball-screw shaker.

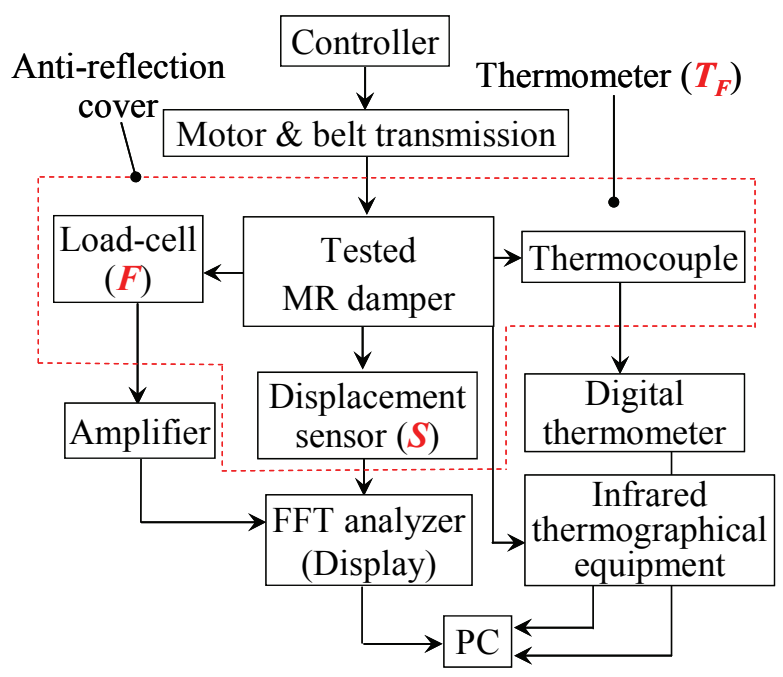

Fig. 3. Loading and measurement equipment attached to the MR damper's endurance test rig. 


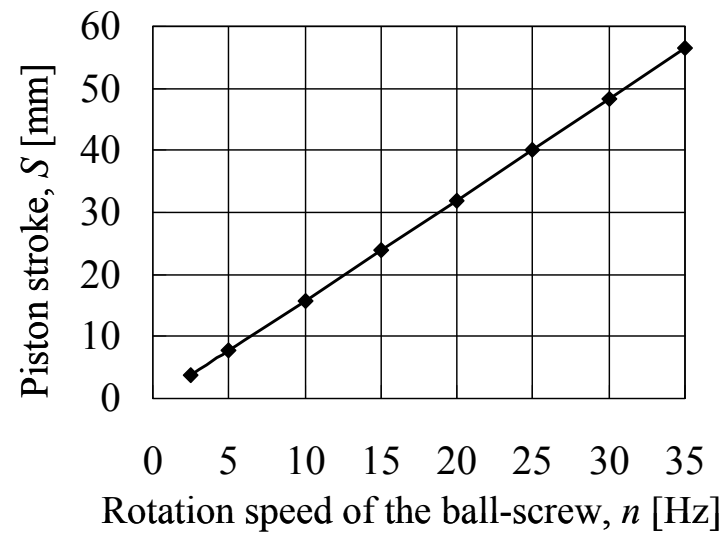

Fig. 4. Variation of the piston stroke versus the rotation speed of the ball-screw shaker.

At augmentation of the number of working cycles, the mechanical energy of vibration transforms into thermal energy, and due to the heat generated inside the MR damper, temperature rise on the external surface of its cylinder can be observed. Such thermal effect is called here "forced heating". On the other hand, following to the endurance test (forced heating), during natural cooling in air of the MR damper for a time interval of 2 hours, temperature drop on the external surface of its cylinder can be observed. Such thermal effect is called here "natural cooling". By using an infrared equipment (Thermo Shot F30W-A01BL), with the measurement wavelength in the range of 8-13 $\mu \mathrm{m}$, the thermographical images were captured and processed.

\subsection{Experimental Procedure}

As Fig. 3 illustrates, after connecting all the elements of the loading and measurement equipment, one performs the setting of the controller, FFT analyzer, and infrared thermographical equipment. Damping force $F$ acting on the piston-rod (load cell); piston stroke $S$ (laser displacement sensor); air temperature inside the anti-reflection cover $T_{F}$ (thermometer); and the temperature $T$ in two points (Fig. 5) on the external surface of the MR damper (thermocouples and digital thermometer) were continuously monitored.

Experimental data was displayed on the screen of the FFT analyzer as hysteresis loops, and also, on the screen of the infrared detection equipment as thermographical photos.

At certain instants, the digital data is saved as ASCII files, readable by MATLAB, which was used to perform the detailed thermal analysis.

MR damper's dissipated energy $E$ is calculated as the area of the hysteresis loop in a $(S, F)$ system of coordinates (see Figs. 6-12 and 20).

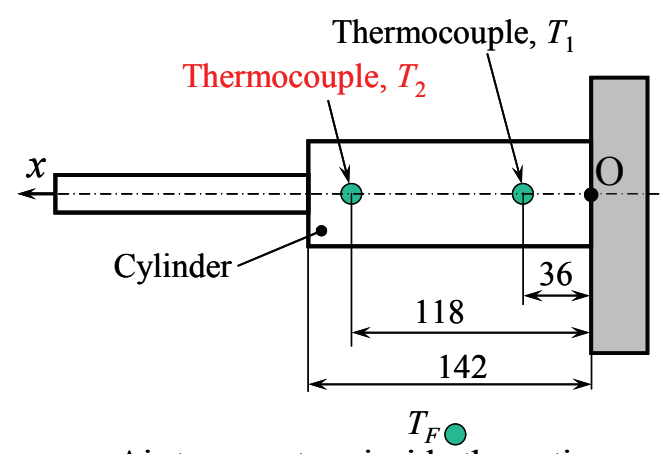

Air temperature inside the antireflection cover (thermometer)

Fig. 5. Location of thermocouples on the external surface of the MR damper's cylinder.

Then, the damping coefficient was calculated as:

$$
C=\frac{2 E}{\pi^{2} f S^{2}} .
$$

Thermal calculus developed in this work is based on the assumptions ${ }^{(2,3)}$ that the heat conduction and the radiant heat transfer can be neglected relative to the natural convective heat transfer of the damper. Thus, the convective infinitesimal heat power $\dot{Q}=d Q / d t \quad$ ( $Q$ is the convective heat) transferred from the cylinder to surroundings in the radial direction, over a small distance $d x$ taken in the axial direction (see Fig. 5), can be written as:

$$
d \dot{Q} / d x=2 \pi R h\left[T(x)-T_{F}\right],
$$

where $R=21 \mathrm{~mm}$ is the radius of the cylinder, and $T(x)$ is the temperature distribution along the axial coordinate of the MR damper. Based on a procedure previously described in Ref. ${ }^{(2,3)}$, mean coefficient of the convective heat transfer $h$ was taken as $20 \mathrm{~W} /\left(\mathrm{m}^{2} \mathrm{~K}\right)$ and as $460 \mathrm{~W} /\left(\mathrm{m}^{2} \mathrm{~K}\right)$ at the interfaces of the cylinder with air and the stainless steel plate (wall), respectively (see Fig. 5).

\section{Experimental Results and Discussions}

\subsection{Hysteresis Loop of the MR Damper}

Variation of the MR damper's hysteresis loop versus the piston stroke for various values of the ball-screw rotation speed $(n=2.5,5,10,15,20,25,30$ and $35 \mathrm{~Hz})$ is shown in Fig. 6 for the control voltage $U=0.0 \mathrm{~V}$, in Fig. 7 for $U=0.4 \mathrm{~V}$, in Fig. 8 for $U=0.8 \mathrm{~V}$, in Fig. 9 for $U=1.2 \mathrm{~V}$, in Fig. 10 for $U=1.6 \mathrm{~V}$, and in Fig. 11 for $U=2.0 \mathrm{~V}$. The same experimental results can be viewed as the variation of the MR damper's hysteresis loop versus the piston stroke 
for various values of the control voltage $(U=0.0,0.4,0.8$, $1.2,1.6$ and $2.0 \mathrm{~V}$ ) and for a certain value of the ball-screw rotation speed, e.g., for $n=35 \mathrm{~Hz}$ in Fig. 12 .

From Figs. 6-12 one observes that the hysteresis loop area, i.e., the MR damper dissipated energy, increases at augmentation of the rotation speed $n$ and control voltage $U$.

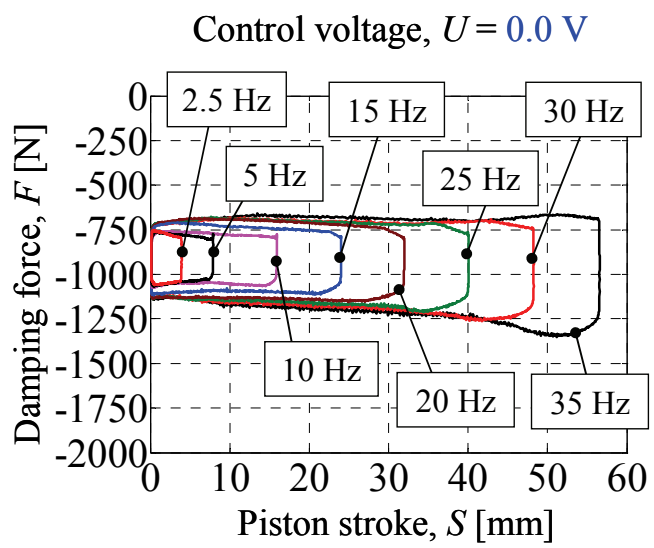

Fig. 6. Variation of the hysteresis loop versus stroke for various rotation speeds of the ball-screw and $U=0.0 \mathrm{~V}$.

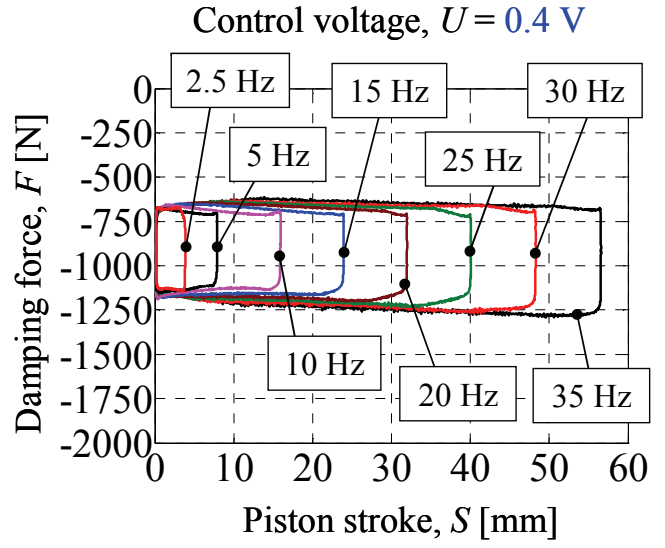

Fig. 7. Variation of the hysteresis loop versus stroke for various rotation speeds of the ball-screw and $U=0.4 \mathrm{~V}$.

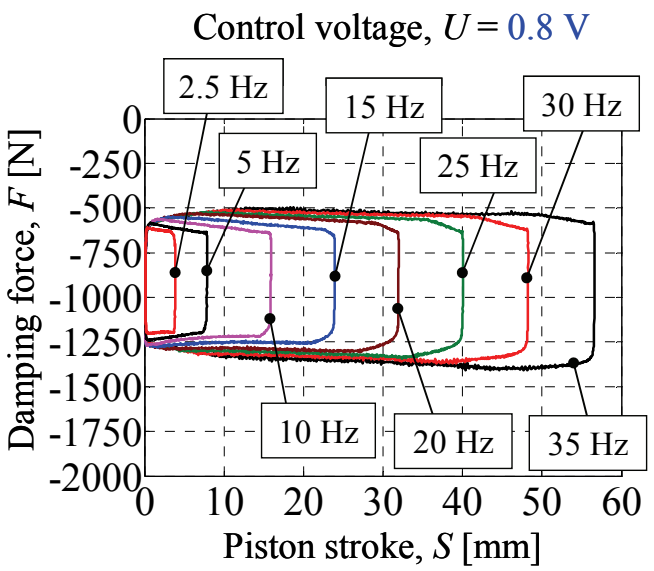

Fig. 8. Variation of the hysteresis loop versus stroke for various rotation speeds of the ball-screw and $U=0.8 \mathrm{~V}$.

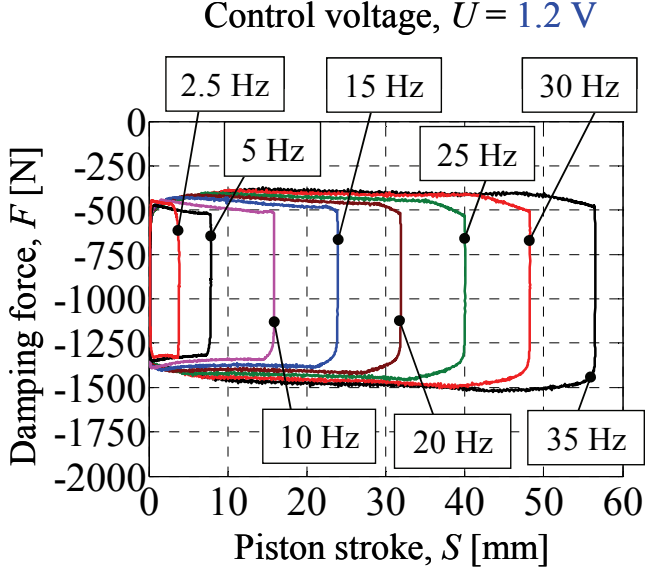

Fig. 9. Variation of the hysteresis loop versus stroke for various rotation speeds of the ball-screw and $U=1.2 \mathrm{~V}$.

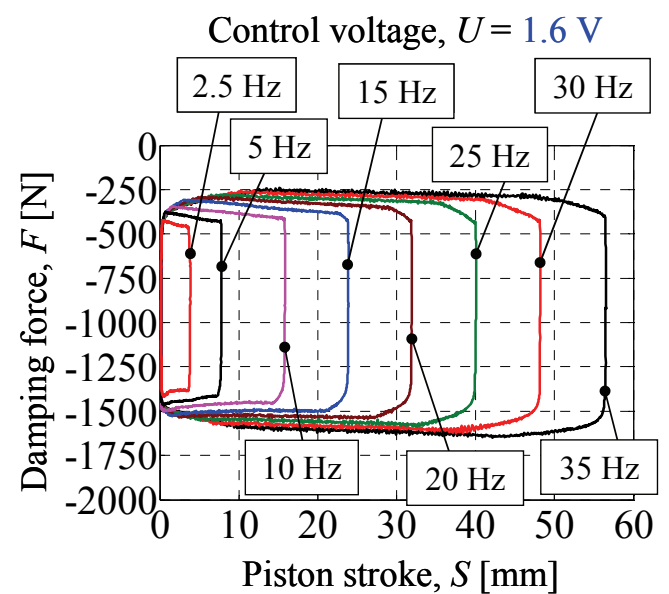

Fig. 10. Variation of the hysteresis loop versus stroke for various rotation speeds of the ball-screw and $U=1.6 \mathrm{~V}$.

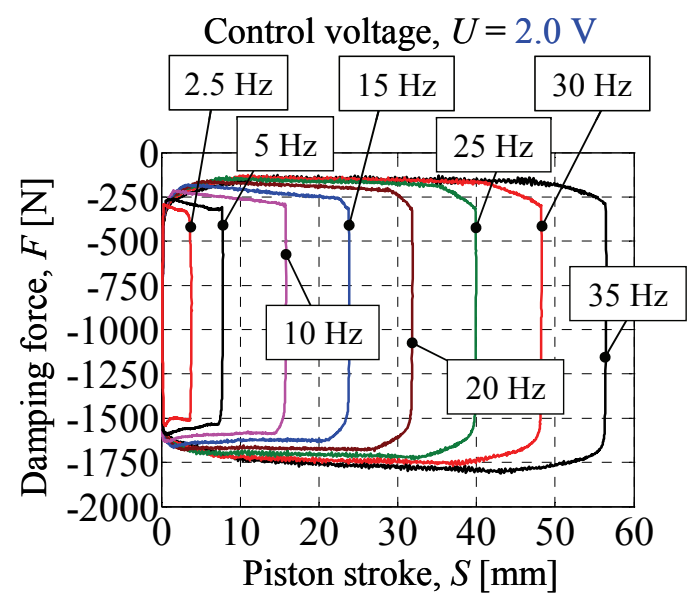

Fig. 11. Variation of the hysteresis loop versus stroke for various rotation speeds of the ball-screw and $U=2.0 \mathrm{~V}$.

Such results are consistent with the well-known fact that, as the magnetic field increases, resistance to fluid flow of the MR colloid increases until the saturation point is reached ${ }^{(6)}$. 


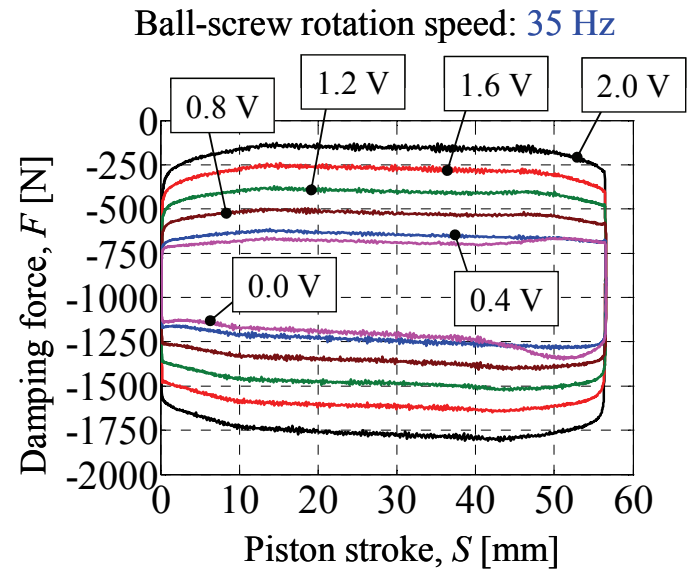

Fig. 12. Variation of the hysteresis loop versus stroke for various values of the control voltage and $n=35 \mathrm{~Hz}$.

\subsection{Dissipative Properties of the MR Damper}

Figures 13 and 14 illustrate the variation of the MR damper's dissipated energy and damping coefficient versus the piston stroke for various values of the control voltage $(U=0.0,0.4,0.8,1.2,1.6$, and $2.0 \mathrm{~V})$.

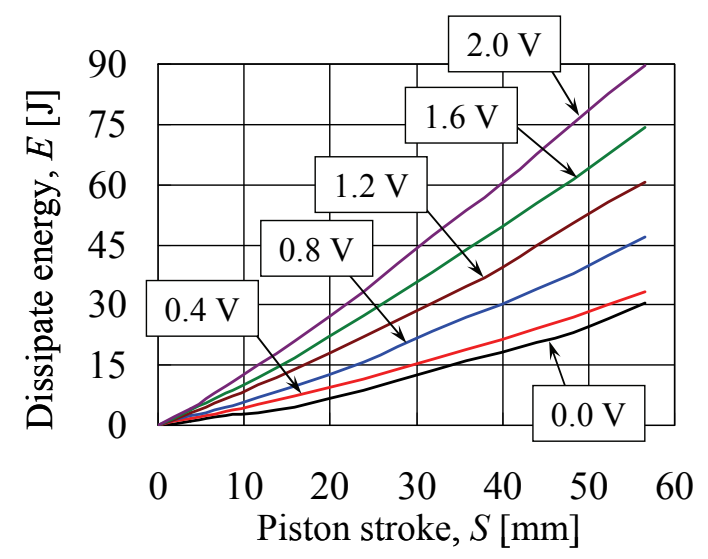

Fig. 13. Variation of the dissipated energy versus the piston stroke for various values of the control voltage.

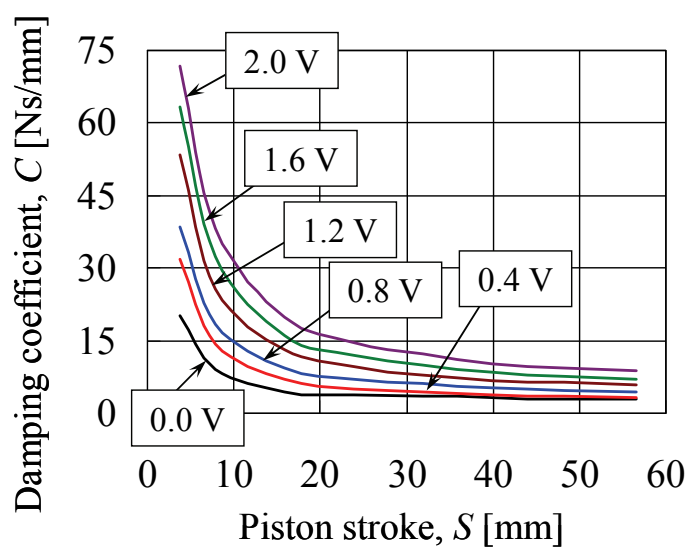

Fig. 14. Variation of the damping coefficient versus the piston stroke for various values of the control voltage.
As expected, dissipated energy increases at augmentation of the piston stroke since the length of the hysteresis loop increases. On the other hand, dissipated energy increases at augmentation of the control voltage $U$ since the width of the hysteresis loop, i.e., the difference between the minimal and the maximal values of the compressive damping force increases.

Oppositely, the damping coefficient $C$ decreases at augmentation of the piston stroke as $1 / S$ type function. Especially for long strokes, exceeding $40 \mathrm{~mm}$, the variation rate of the damping coefficient versus $S$ becomes negligible. On the other hand, as expected, the damping coefficient increases at augmentation of the control voltage $U$. Thus, especially in the region of long strokes, where the damping coefficient displays a flat graph against the piston stroke, the control through a variable magnetic field of the MR damper might have interesting practical applications.

In conclusion, results shown in Figs. 13 and 14 prove the ability of the evaluated MR damper to be used for achieving intelligent automotive suspensions. Such conclusion is also supported by the fact that the response time of the MR fluid is shorter than $15 \mathrm{~ms}$ relative to the applied change in the magnetic field, and also by the fact that the control is achieved under low voltages, usually available in the electric circuit of an autovehicle.

\subsection{Thermographical Images of the MR Damper}

Figure 15 presents some illustrative thermographical images of the external surface of the tested long-stroke MR damper.

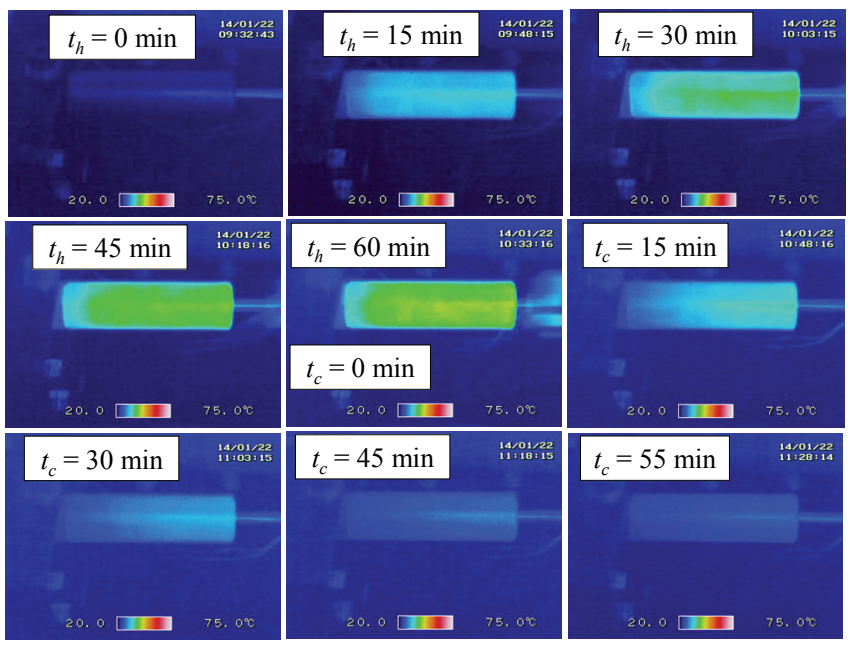

Fig. 15. Thermographical images of the MR damper's external surface, recorded during forced heating at the instants $t_{h}=0,15,30,45$ and $60 \mathrm{~min}$, and then during natural cooling at the instants $t_{c}=0,15,30,45$ and $55 \mathrm{~min}$. 
Such thermographical images were recorded during forced heating at the instants $t_{h}=0,15,30,45$ and $60 \mathrm{~min}$, and then, during natural cooling in air at the instants $t_{c}=0,15$, 30, 45 and $55 \mathrm{~min}$. Pictures displayed for the last instant of forced heating $\left(t_{h}=60 \mathrm{~min}\right)$ and for the first instant of natural cooling $\left(t_{c}=0\right)$ are the same. One observes that the heat is generated towards the right side of the cylinder, due to the effect of fluid friction, occurring when the MR colloid passes through the valves and orifices of the piston head, and also due to the effect of dry friction, occurring between the piston-rod and the seals. On the other hand, the left side of the cylinder is cooler, due to the presence of the nitrogen tank and the vertical plate used to fix the MR damper on the test rig (see also Fig. 1).

\subsection{Validation of the Thermographical Results}

In order to evaluate the accuracy of the temperature distributions obtained by processing the thermographical images, one firstly should achieve a base of reference.

To this end, Figure 16 presents the variation of the temperatures $T_{1}$ and $T_{2}$ (recorded by using thermocouples, see Fig. 5), as well as the environmental temperature $T_{F}$ (recorded by using a thermometer) versus the elapsed time $t$. Such endurance test was performed during 1 hour for a ball-screw speed of $n=35 \mathrm{~Hz}$. In these circumstances, the actual piston stroke was $S=56.5 \pm 0.07 \mathrm{~mm}$, and the actual excitation frequency was $f=0.66 \pm 0.005 \mathrm{~Hz}$. Control voltage was set to $U=0.0 \mathrm{~V}$. Forced heating experiment was followed by natural cooling in air, for a total period of 3.5 hours. One observes that during excitation test, due to the heating of the test rig, environmental temperature inside the anti-reflection cover increases.

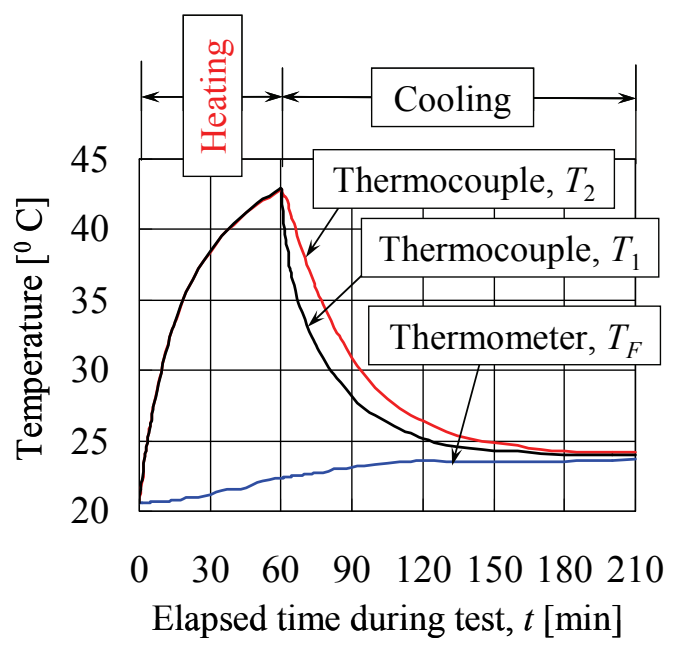

Fig. 16. Variation of the temperatures $T_{1}, T_{2}$ (recorded by thermocouples) and environmental temperature $T_{F}$ (recorded by thermometer) versus the elapsed time $t$.

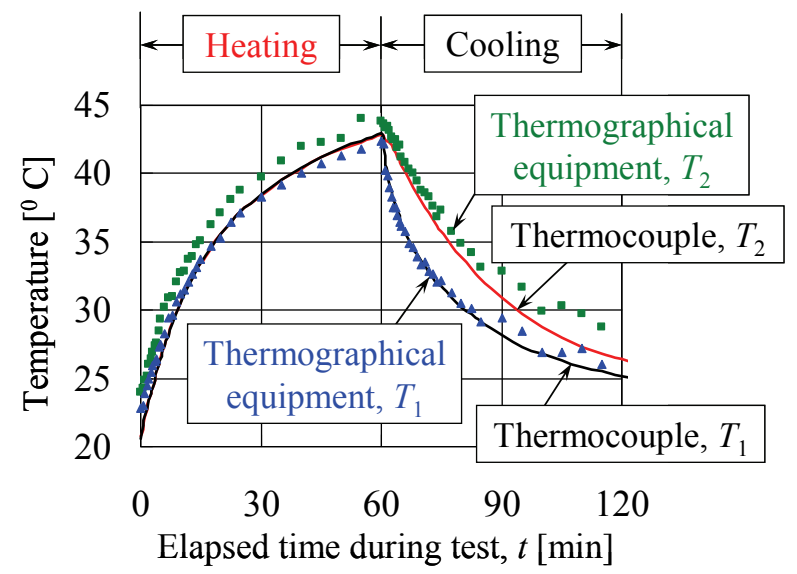

Fig. 17. Variation of the temperatures $T_{1}, T_{2}$ (recorded by thermocouples and obtained by processing the thermographical images) versus the elapsed time $t$.

Concerning the variation of $T_{1}$ and $T_{2}$, there is no significant difference between them during forced heating, but higher speed of cooling can be observed for thermocouple $T_{1}$, which is placed in closer proximity to nitrogen tank and also to vertical plate used to fix the MR damper on the test rig (see again Figs. 1 and 5). As expected, at the end of the natural cooling process there is no significant difference between temperatures $T_{1}, T_{2}$ and $T_{F}$.

Next, one compares the temperatures obtained by using the thermocouples $T_{1}$ and $T_{2}$ with those obtained by processing the thermographical images of the MR damper (see Fig. 17). One observes quite good agreement between the results obtained through the thermographical technique and the results obtained through classical method (thermocouple and digital thermometer), especially for the temperature $T_{1}$.

Taking as reference the results given by thermocouples, the relative error is smaller than $6 \%$ in the case of temperature $T_{1}$, and smaller than $10 \%$ in the case of temperature $T_{2}$, for the whole period of elapsed time. In conclusion, with a margin for error of $10 \%$, thermographical method provides quite accurate results concerning the temperature distributions.

\subsection{Temperature Distributions on the Cylinder}

Temperature distributions $T$ on the MR damper's external surface along the longitudinal axis $x$ are illustrated at various instants $t_{h}=0,1.5,2.5,3,5,6,8,10,12,15,20,25$, 30, 40, 50 and $60 \mathrm{~min}$ during endurance tests (see the forced heating process in Fig. 18), as well as at instants $t_{c}=$ $0,1,2,3,5,7,9,12,15,20,25,30,35,45$ and $55 \mathrm{~min}$ during natural cooling in air (see Fig. 19). 


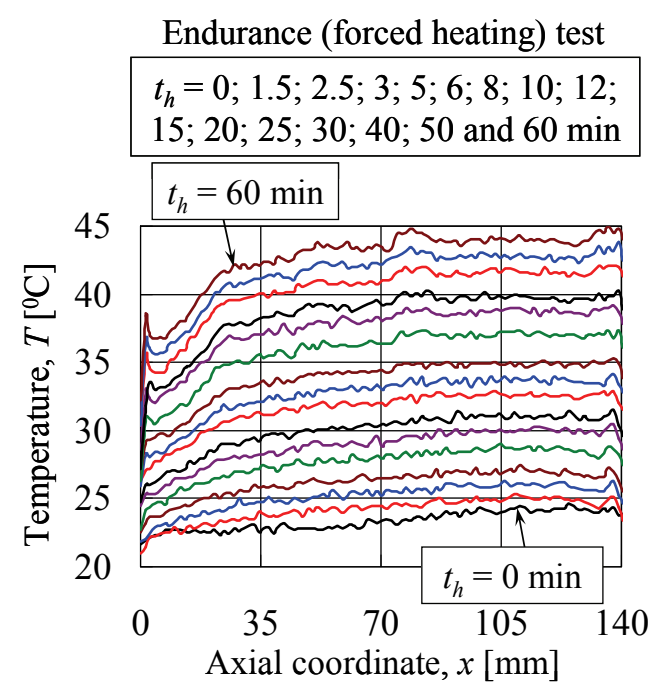

Fig. 18. Variation of the temperature $T$ on the external surface of the MR damper along the longitudinal axis $x$, measured during endurance (forced heating) tests.

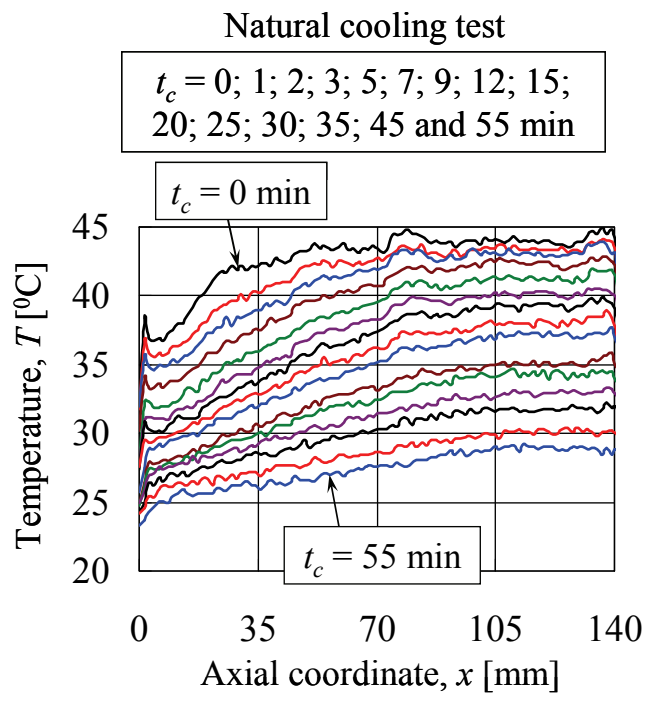

Fig. 19. Variation of the temperature $T$ on the external surface of the MR damper along the longitudinal axis $x$, measured during natural cooling in air.

Curve displayed in Fig. 18 for the last instant of forced heating $\left(t_{h}=60 \mathrm{~min}\right)$ and the curve shown in Fig. 19 for the first instant of natural cooling $\left(t_{c}=0\right)$ are the same. Again, one observes that heat is generated towards the right side of the cylinder, due to the combined effects of fluid and dry friction. On the other hand, the left side of the cylinder is cooler, due to the presence of the nitrogen tank and the vertical plate used to fix the MR damper on the test rig.

\subsection{Dissipated Energy and Generated Heat}

Figure 20 illustrates the variation of the hysteresis loop versus the elapsed time during the endurance test $\left(t_{h}=0,0.5\right.$,
$3.5,6,25$ and $60 \mathrm{~min}$ ), for a control voltage $U=0 \mathrm{~V}$ and a ball-screw speed of $n=35$. From Fig. 20 one observes that the hysteresis loop largely changes against the elapsed time and this is induced by the continuous temperature variation.

Next, dissipated energy $E$ is calculated as the area of the hysteresis loops shown in Fig. 20, and the generated heat is evaluated by integrating Eq. (2) over the whole external surface of the MR damper. Thus, Fig. 21 illustrates the variation of the dissipated energy and generated heat versus the elapsed time during endurance (forced heating) test.

From Fig. 21 one observes that at the beginning of the endurance test, the dissipated energy significantly decreases and then reaches a minimum value in the interval of $t_{h}=$ 20-25 min. This effect is induced by the decreasing of the MR fluid's viscosity at the temperature augmentation. After that, the energy dissipated slightly increases in the interval of $t_{h}=25-60 \mathrm{~min}$, and such effect is more likely to be induced by the increase of the dry friction at the seals under temperature augmentation. On the other hand, the generated heat monotonically increases versus the elapsed time, and finally tends to approach the value of the dissipated energy.

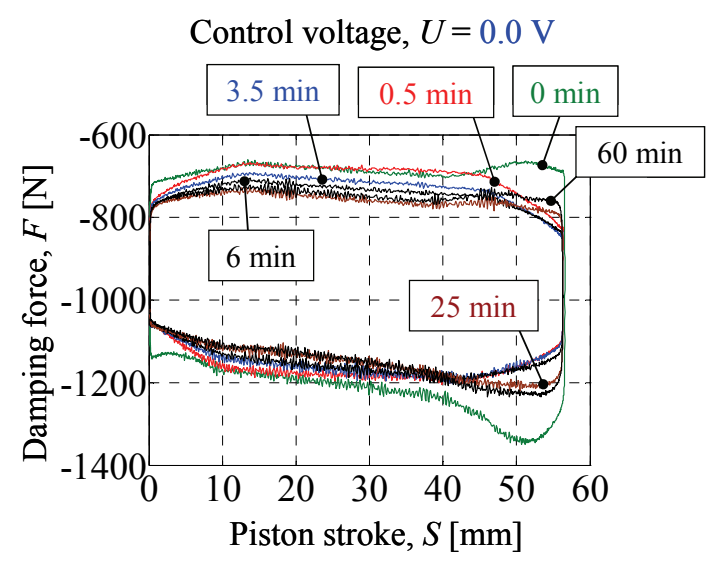

Fig. 20. Variation of the hysteresis loop versus the elapsed time during the endurance test for $U=0 \mathrm{~V}$ and $n=35 \mathrm{~Hz}$.

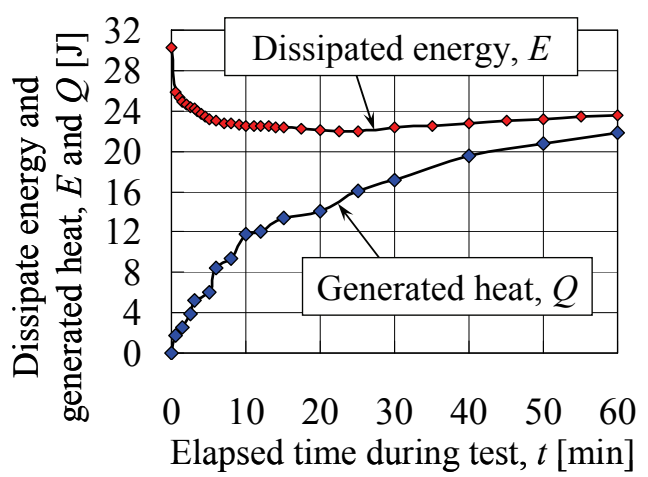

Fig. 21. Variation of the dissipated energy and generated heat versus the elapsed time during the endurance test. 
Thus, under the circumstances that an almost stationary thermal regime was attained, i.e., for $t_{h}$ approaching $60 \mathrm{~min}$, the generated heat curve tends to come nearer to the dissipated energy curve.

\section{Conclusions}

In this work the dissipative and thermal characteristics of a monotube long strong magneto-rheological (MR) damper, to be employed for automotive suspension, were evaluated. The following main conclusions were inferred:

1) Area of the hysteresis loop, i.e., the MR damper dissipated energy increases at augmentation of the rotation speed of the ball-screw shaker, and the control voltage. Such results are consistent with the well-known facts that longer piston stroke is obtained at augmentation of the rotation speed, and larger resistance to fluid flow of the MR colloid is obtained as the magnetic field increases.

2) Damping coefficient decreases at augmentation of the piston stroke as an inverse-type function. Especially for long strokes, exceeding $40 \mathrm{~mm}$, the variation rate of the damping coefficient versus stroke becomes negligible. On the other hand, damping coefficient increases at augmentation of the control voltage. Thus, especially in the region of long strokes, where the damping coefficient displays a flat graph against the piston stroke, the control through a variable magnetic field of the MR damper might have interesting practical applications.

3) Based on the ability to control the dissipated energy and damping coefficient, one concludes that the evaluated MR damper can be used to achieve intelligent automotive suspensions. Such conclusion is also supported by the fact that the response time of the MR fluid is shorter than $15 \mathrm{~ms}$ relative to the applied change in the magnetic field, and also by the fact that the control is achieved under low voltages, usually available in the electric circuit of an autovehicle.

4) With a margin for error of $10 \%$, thermographical method provides quite accurate results concerning the temperature distributions recorded on the external surface of the MR damper's cylinder.

5) MR damper's hysteresis loop largely changes against the elapsed time during endurance (forced heating) experiments, and this effect seems to be induced by the continuous temperature variation.

Thus, at the beginning of the endurance test, the dissipated energy significantly decreases and then reaches a minimum value in the interval of $t_{h}=20-25 \mathrm{~min}$. This effect is induced by the decreasing of the MR fluid's viscosity at the temperature augmentation. After that, the dissipated energy slightly increases in the interval of $t_{h}=25-60 \mathrm{~min}$, and such effect is more likely to be induced by the increase of the dry friction at the seals under temperature augmentation.

6) Generated heat monotonically increases versus the elapsed time, and finally tends to approach the value of the dissipated energy. Thus, under the circumstances that an almost stationary thermal regime was attained, i.e., for $t_{h}$ approaching $60 \mathrm{~min}$, the generated heat curve tends to come nearer to the dissipated energy curve.

7) Results obtained in this work together with the data provided by MR damper manufacturer are useful for the appropriate design of intelligent automotive suspensions.

\section{Acknowledgments}

This research was supported by the Japanese Ministry of Education, Grant-in-aid for scientific fundamental research, Project C-25420101. Authors would like to acknowledge the technical support of Mr. Shunsuke Ohga to achieve reliable experimental results.

\section{References}

(1) Seiyed Hamid Zareh, Atabak Sarrafan, Amir Ali Akbar Khayyat, and Abolghassem Zabihollah : "Intelligent Semi-Active Vibration Control of Eleven Degrees of Freedom Suspension System using Magnetorheological Dampers", Journal of Mechanical Science and Technology, Vol. 26, Issue 2, pp. 323-334, 2012

(2) Valentin Suciu, Shingo Tani, and Kenta Miyoshi : "Experimental Study on the Thermal Characteristics of a Colloidal Damper", Journal of System Design and Dynamics, Vol. 4, No. 6, pp. 899-913, 2010

(3) Valentin Suciu, and Yuta Kimura : "Experimental Study on the Forced Heating and Natural Cooling of a Colloidal Damper", Journal of System Design and Dynamics, Vol. 6, No. 5, pp. 538-554, 2012

(4) *** : "LORD Technical Data, RD 8040-1 and RD 8041-1 Dampers", LORD Corporation, OD DS7016, pp. 1-2, 2009

(5) *** : "LORD Wonder Box Device Controller Kit", LORD Corporation, OD U17000, pp. 1-2, 2006

(6) L. Liggieri, A. Sanfeld, and A. Steinchen, "Effects of Magnetic and Electric Fields on Viscosity and Surface Tension of Liquids", Physica A, Vol. 206, pp. 299-331, 1994 